\title{
Advanced therapy for pulmonary arterial hypertension due to congenital heart disease: a clinical perspective in a new therapeutic era
}

\author{
M. J. Schuuring • S. M. Boekholdt • A. Windhausen • B. J. Bouma • M. Groenink • \\ M. Keijzers • R. J. De Winter • D. R. Koolbergen • N. A. Blom • B. J. M. Mulder
}

Published online: 16 November 2011

(C) The Author(s) 2011. This article is published with open access at Springerlink.com

\section{Introduction}

Pulmonary arterial hypertension (PAH) is a progressive disease with poor survival. PAH is classified by the 2009 updated clinical classification of pulmonary hypertension and a major subgroup is PAH due to congenital heart disease (CHD) with systemic-to-pulmonary shunt [1]. This shunting may lead to extensive histological changes in the distal pulmonary arteries resulting in an irreversible increase in pulmonary vascular resistance (PVR). The classification of CHD-PAH includes four categories: (1) Eisenmenger syndrome, (2) PAH associated with systemicto-pulmonary shunts, (3) PAH with small septal defects,

\footnotetext{
M. J. Schuuring $\cdot$ S. M. Boekholdt $\cdot$ A. Windhausen •

B. J. Bouma $\cdot$ M. Groenink $\cdot$ R. J. De Winter

B. J. M. Mulder $(\square)$

Department of Cardiology, Academic Medical Center,

PO Box 22660, 1100 DD, Amsterdam, the Netherlands

e-mail: b.j.mulder@amc.uva.nl
}

M. J. Schuuring • B. J. M. Mulder

Interuniversity Cardiology, Institute of the Netherlands,

Utrecht, the Netherlands

\section{Keijzers}

Department of Cardiology, Kennemer Gasthuis,

Haarlem, the Netherlands

\section{R. Koolbergen}

Department of Cardio-thoracic Surgery,

Academic Medical Center,

Amsterdam, the Netherlands

N. A. Blom

Department of Paediatric Cardiology,

Leiden University Medical Center,

Leiden, the Netherlands and (4) PAH after corrective cardiac surgery [2]. In these patients advanced pharmacological therapy should be considered.

Three classes of advanced therapy for PAH are currently in use: prostanoids such as epoprostenol, endothelin-1 receptor antagonists such as bosentan, and phosphodiesterase 5 inhibitors such as sildenafil [3]. Bosentan has been shown to improve 6-min walking distance (6MWD) and to decrease PVR in patients with Eisenmenger syndrome [4]. Anecdotal experience with the phosphodiesterase type-5 inhibitors sildenafil and tadalafil show favourable functional and haemodynamic results in patients with CHD-PAH $[5,6]$. We present three patients treated with advanced pharmacological therapy for CHD-PAH: a patient with Eisenmenger syndrome receiving standard care, a patient with atrial septal defect receiving advanced therapy as a bridge to surgery, and a patient with segmental PAH who was started on advanced therapy empirically.

\section{Case 1: an Eisenmenger patient}

The first case describes a 38-year-old male patient with a double inlet left ventricle, hypoplastic aortic arch and patent ductus arteriosus. He was deemed ineligible for a Fontan circulation and developed PAH in childhood. He was severely symptomatic (NYHA functional class III-IV) and could only live a sedentary lifestyle. He had been on the waiting list for combined heart and lung transplantation for 2 years. He was cyanotic with a peripheral saturation of $80 \%$. His haemoglobin level was $13.4 \mathrm{mmol} / \mathrm{L}$. On auscultation, normal heart sounds were heard, as well as a systolic murmur grade II/VI. Trans-thoracic echocardiography (TTE) showed a moderate systolic ventricular function, mild mitral regurgitation, and moderate tricuspid regurgita- 
tion (Fig. 1). His 6MWD was $475 \mathrm{~m}$, and during exercise his saturation dropped from $80 \%$ to $62 \%$

It was decided to initiate bosentan $125 \mathrm{mg}$ twice daily, as monotherapy. He improved to NYHA functional class II and his six 6MWD increased from 475 to 500, 517 and $557 \mathrm{~m}$ at 1, 2 and 3 years, respectively. His saturation remained unchanged. Clinically he has improved over the past 4 years on bosentan monotherapy and is now able to work $12 \mathrm{~h}$ a week. He could be removed from the transplantation list.

\section{Case 2: advanced therapy as a bridge to atrial septal defect closure}

A 25-year-old female patient was referred to our hospital because of progressive dyspnoea for 5 months. Her medical history was unremarkable, and auscultation revealed fixed splitting of the second heart sound. The ECG showed sinus tachycardia of 120 beats/min, right heart axis, and tall $\mathrm{R}$ waves in V1 and V2. The chest x-ray showed a dilated right pulmonary artery and pulmonary trunk. A CT scan was performed to rule out pulmonary embolism. Pulmonary embolism was not observed, but the right ventricle and pulmonary trunk were severely dilated. In addition, an anomalous connection of the right upper pulmonary vein to the superior caval vein was found. TTE revealed a small left ventricle with good systolic function. The right ventricle was dilated with poor systolic function. The interventricular septum was flattened during both diastole and systole, suggesting right-sided pressure overload. Agitated saline contrast injection revealed shunting from the right to left atrium, suggestive of an atrial septal defect. Transoesophageal echocardiogram (TEE) showed a sinus venosus defect with a right-to-left shunt (Fig. 2). Right

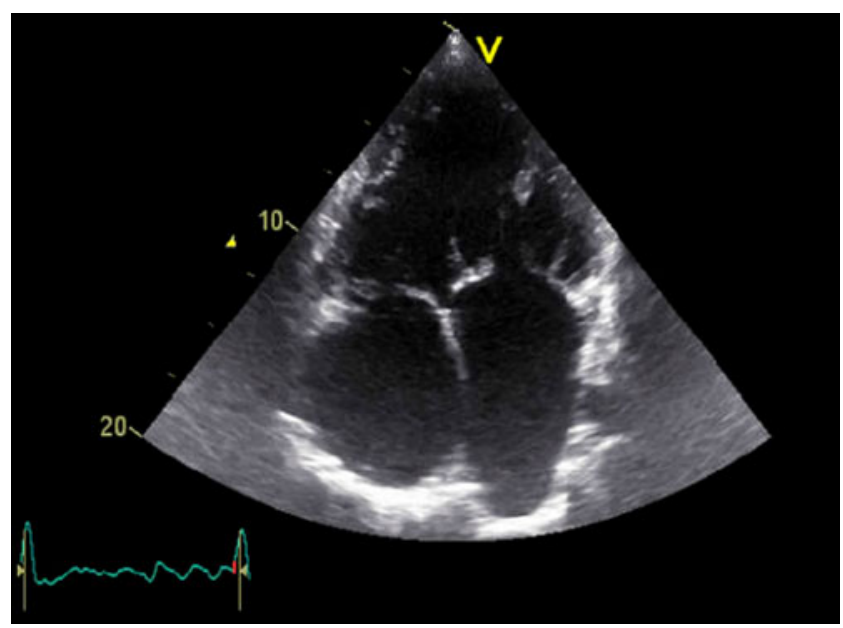

Fig. 1 Eisenmenger patient in case 1. Transthoracic echocardiogram demonstrating double inlet left ventricle heart catheterisation demonstrated a pulmonary arterial pressure (PAP) of $89 / 46 \mathrm{mmHg}$ (mean $55 \mathrm{mmHg}$ ) with a systemic blood pressure of $118 / 65 \mathrm{mmHg}$ (mean $82 \mathrm{mmHg}$ ). PVR was markedly elevated at 680 dynes/ $\mathrm{s} / \mathrm{cm}^{-5}$ (8.5 Wood Units). Epoprostenol infusion to achieve maximal pulmonary vascular dilation did not result in a reduction of PVR. Her baseline 6MWD was $514 \mathrm{~m}$ and saturation dropped from $97 \%$ at rest to $78 \%$ during exercise. The working diagnosis at that time was PAH associated with systemic-to-pulmonary shunt (Group 2). However, given the unusually severe PAH for a patient with sinus venosus defect at this age, we could not rule out that the clinical picture was explained by a combination of idiopathic pulmonary arterial hypertension (IPAH, formerly called primary pulmonary hypertension) and a sinus venosus defect.

This patient was treated with combination therapy of bosentan $125 \mathrm{mg}$ twice daily and sildenafil $20 \mathrm{mg}$ three times daily. After several weeks of therapy, subjective improvement was observed. After 3 months of treatment, repetition of the right heart catheterisation showed that the PAP had dropped to $62 / 32 \mathrm{mmHg}$ (mean $42 \mathrm{mmHg}$ ) with a systemic blood pressure of $104 / 54 \mathrm{mmHg}$ (mean $73 \mathrm{mmHg}$ ). In addition, her 6MWD increased from 514 to $640 \mathrm{~m}$, NT-pro-BNP decreased from 1092 to $497 \mathrm{ng} / \mathrm{L}$, and saturation during exercise increased from $78 \%$ to $90 \%$. Because of the beneficial response to medical therapy, this patient was planned for closure of the sinus venosus defect and rerouting of the pulmonary vein flow to the left atrium. The interatrial septum will be reconstructed with a fenestrated patch to facilitate complete closure via percutaneous intervention at a later stage. This 'staged' procedure was chosen to minimise the risk of acute right-sided heart failure, which is a life-threatening complication.

\section{Case 3: advanced therapy in a patient with segmental PAH started empirically}

The third case describes a 39-year-old female patient who presented with shortness of breath. She had a history of pulmonary atresia, ventricular septal defect and stenosis of a major aortopulmonary collateral artery (MAPCA) to the right inferior pulmonary lobe (Fig. 3). In childhood she had initially been palliated with a left-sided Blalock-Taussig shunt to supply pulmonary blood flow, and later with a central Goretex shunt. New cardiac evaluation followed in adolescence. At that time further surgical repair was not indicated, because of her stable cardiac situation and the risk of complications associated with a reoperation. The pulmonary lobes supplied by the aorta-pulmonary shunt or the non-stenosed MAPCAs experienced a systemic blood pressure and developed $\mathrm{PAH}$, whereas the right inferior 
A

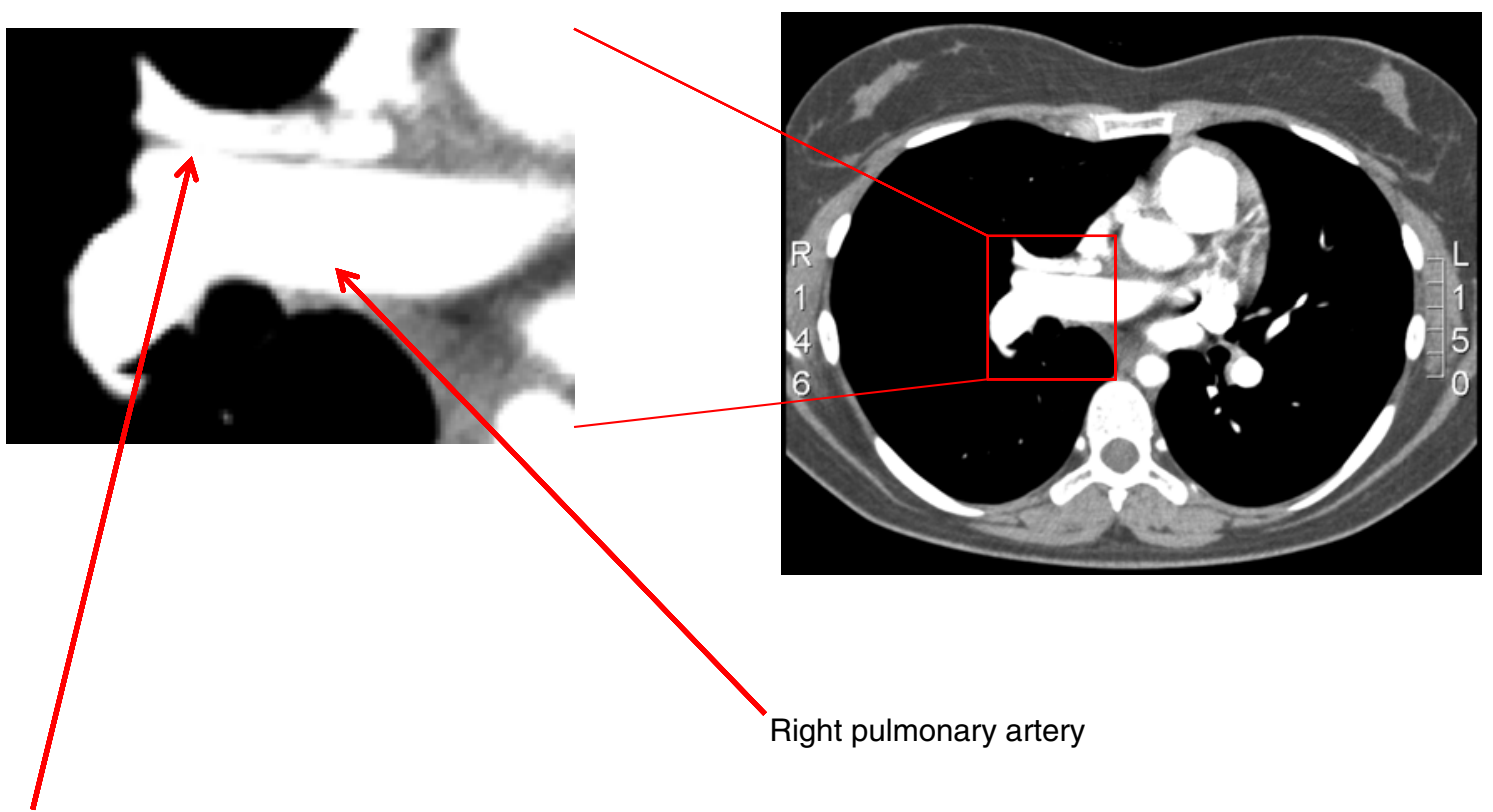

Anomalous connection of the right upper pulmonary vein to the superior caval vein

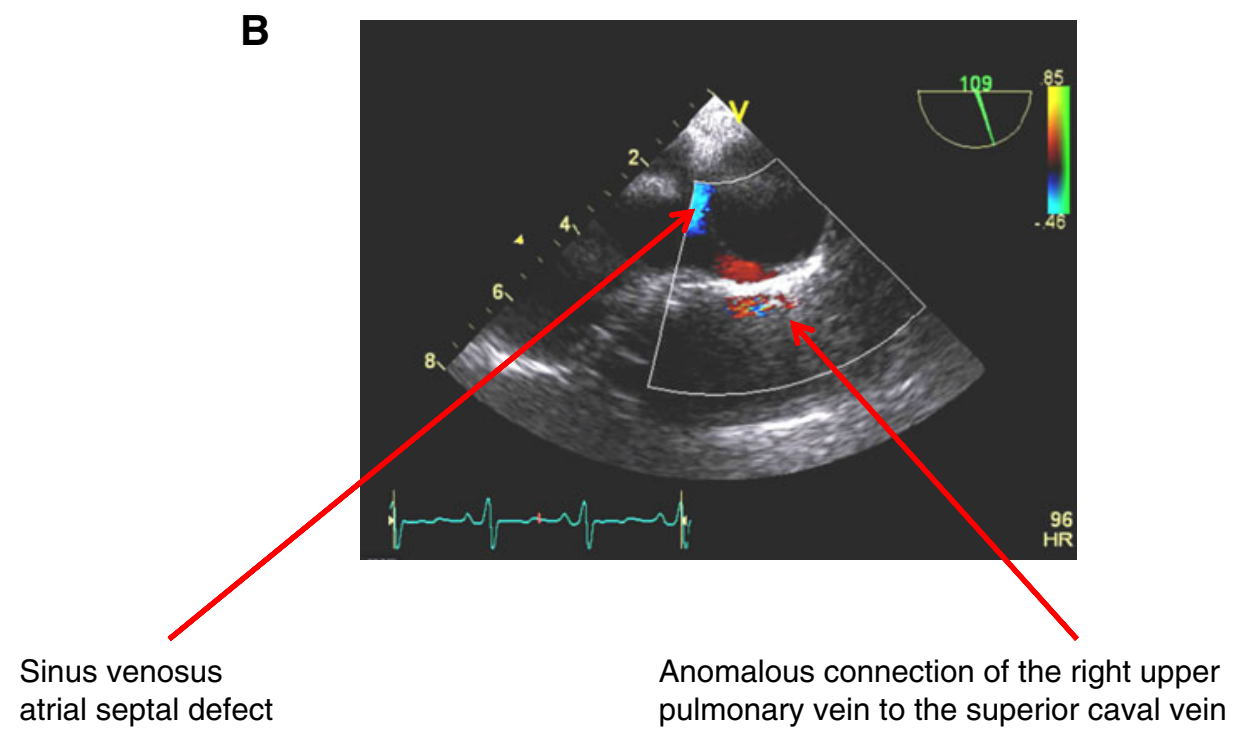

Fig. 2 Sinus venosus atrial septal defect in case 2. a Cardiac computed tomography scan, b Transoesophageal echocardiogram

lobe supplied by the stenosed MAPCA experienced a relatively normal PAP and as a consequence did not develop PAH. The term segmental PAH has been suggested to describe this situation. She was cyanotic with a peripheral saturation of $81 \%$. No pathological murmurs were heard at auscultation. Her NT-pro-BNP was $419 \mathrm{ng} / \mathrm{L}$. Echocardiographically, her right and left ventricular systolic function were good. Her 6MWD was $462 \mathrm{~m}$ and saturation dropped from $81 \%$ to $65 \%$ at exercise.
Because of her shortness of breath, it was decided to treat this patient empirically with bosentan $125 \mathrm{mg}$ twice daily as monotherapy. After initiation, clinical improvement was observed. Her 6MWD increased from 462 to 478, 502 and $513 \mathrm{~m}$ at 1,2 and 3 years respectively. After 3 years of treatment saturation at rest increased to $85 \%$, and NT-pro-BNP decreased to $334 \mathrm{ng} / \mathrm{L}$. Clinically she improved to mild shortness of breath. She experiences fatigue occasionally. 
Fig. 3 Segmental pulmonary arterial hypertension in case 3 . a Magnetic resonance angiography demonstrating left inferior MAPCA stenosis, b Schematic drawing
A

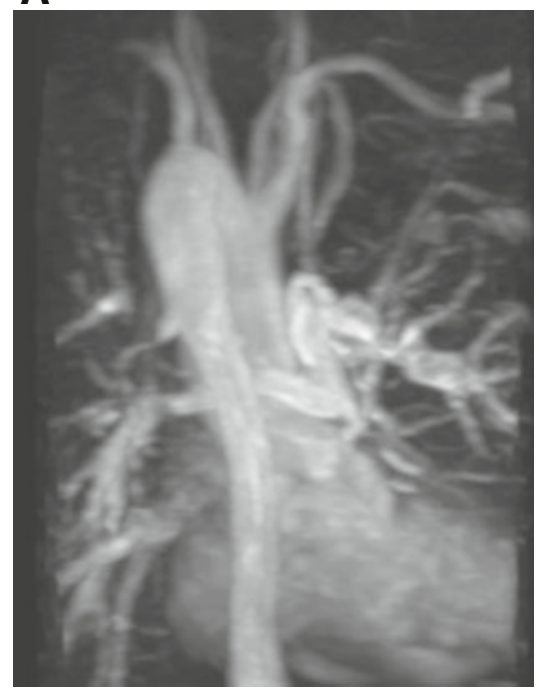

Posterior view
B

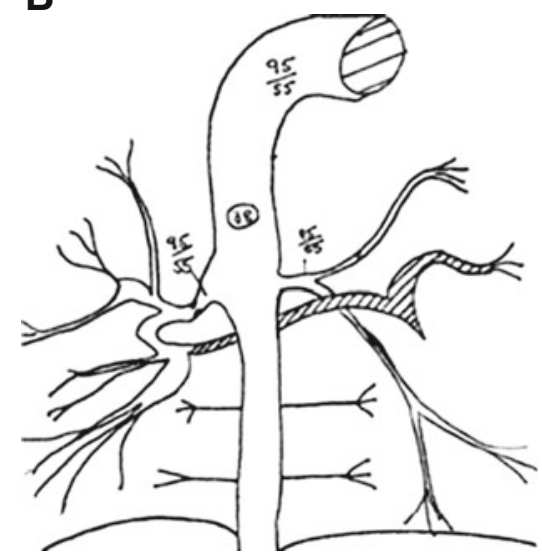

Anterior view

\section{Discussion}

We describe three adults where advanced pharmacological therapy for CHD-PAH was prescribed in different clinical settings. The first patient, diagnosed with Eisenmenger syndrome, was treated according to current guidelines [7]. In the second patient, therapy was started more aggressively as a bridge to atrial septal defect closure. The third patient with segmental PAH was started on advanced therapy empirically.

Presentation with Eisenmenger syndrome, as in the first patient, has a negative impact on survival. Eisenmenger syndrome patients are highly symptomatic, and require major lifestyle adjustments due to limited functional capacity. Quality of life is diminished and pregnancy is strictly contraindicated [8]. In our extended experience with a nationwide standardised protocol on advanced treatment in CHD-PAH, a prolonged beneficial effect of bosentan treatment on 6MWD, echocardiographic stroke volume and quality of life in patients with Eisenmenger syndrome has been shown [9]. In patients with additional Down syndrome stabilisation was seen [9]. The 6MWD is associated with clinical outcome and prognosis [10]. In the second patient optimisation with advanced therapy led to eligibility for surgical closure. The decision to proceed with surgery was made, regardless of the persistent high mean PAP, on the basis of reversibility in PVR after 3 months of advanced medical treatment. Patients with septal defects initially ineligible for cardiac surgery due to severe PAH could benefit from advanced therapy as a bridge to surgical intervention. Patients with segmental PAH, such as our patient in case three, are rare. Segmental PAH is often due to post-surgical pulmonary artery stenosis, resulting in local differences in pulmonary artery pressure and severity of the pathophysiological changes in the pulmonary circulation. Our group has reported on several cases with segmental $\mathrm{PAH}$ demonstrating improvement of NYHA functional class and exercise capacity after 12 months of bosentan treatment [11].

Also in other patient categories, evidence on beneficial effect of advanced therapy is increasing. In NYHA functional class II PAH patients, short-term efficacy of bosentan has been demonstrated [12]. However, this study included only 16 patients with CHD-PAH.

Another group of patients who might benefit from advanced therapy are those with a Fontan circulation. In Fontan patients' plasma endothelin-1 level has been shown to be elevated [13]. Endothelin-1 induces pulmonary vasoconstriction, and increases PVR. Low PVR is crucial for a well-functioning Fontan circulation, and endothelin-1 receptor antagonists might therefore contribute to improvement of clinical status in these patients. We are currently performing a prospective randomised trial on bosentan in Fontan patients; the results are expected in spring 2012 [14].

Finally, advanced therapy might be useful in CHD patients who undergo cardiac surgery, who tend to have a decline in right ventricular function [15]. Treatment with an endothelin-1 receptor antagonist might reduce perioperative pulmonary vasoconstriction induced by the endothelin-1 release initiated by the cardiopulmonary bypass [16]. In a small study, perioperative treatment with a selective endothelin-1 receptor antagonist led to a significant decrease in PVR compared with the control group [17]. However, trials are needed to confirm this hypothesis and to show clinical benefit in CHD patients. In our centre we are 
currently evaluating the effect of bosentan on perioperative right ventricular function prospectively.

\section{Conclusion}

CHD-PAH remains a severe disease, leading to reduced quality of life and cardiac complications including right ventricular failure, arrhythmias and premature death. Our cases demonstrate the variety of indications in which advanced therapy is justified. Research is warranted in CHD patients with elevated endothelin-1 levels, who are at risk of increase in pulmonary artery pressure, to determine whether these patients benefit of advanced therapy.

Open Access This article is distributed under the terms of the Creative Commons Attribution Noncommercial License which permits any noncommercial use, distribution, and reproduction in any medium, provided the original author(s) and source are credited.

\section{References}

1. Duffels MGJ, Engelfriet PM, Berger RMF, et al. Pulmonary arterial hypertension in congenital heart disease: an epidemiologic perspective from a Dutch registry. Int J Cardiol. 2007;120(2):198-204.

2. Simonneau G, Robbins IM, Beghetti M, et al. Updated clinical classification of pulmonary hypertension. J Am Coll Cardiol. 2009;54(1 Suppl):S43-54.

3. Schuuring MJ, Vis JC, Duffels MG, et al. Adult patients with pulmonary arterial hypertension due to congenital heart disease: a review on advanced medical treatment with bosentan. Ther Clin Risk Manag. 2010;6:359-66.

4. Galiè N, Beghetti M, Gatzoulis M, et al. Bosentan Randomized Trial of Endothelin Antagonist Therapy-5 (BREATHE-5) Investigator. Bosentan therapy in patients with Eisenmenger Syndrome: a multicenter, double-blind, randomized, placebo-controlled study. Circulation. 2006;114:48-54.
5. Palazzini M, Manes A, Romanazzi S, et al. Effects of sildenafil treatment in patients with pulmonary hypertension associated with congenital cardiac shunts. Eur Heart J. 2007;28:308.

6. Mukhopadhyay S, Sharma M, Ramakrishnan S, et al. Phosphodiesterase-5 inhibitor in Eisenmenger syndrome: a preliminary observational study. Circulation. 2006;114(17):1807-10.

7. Galiè N, Hoeper MM, Humbert M, et al. Guidelines for the diagnosis and treatment of pulmonary hypertension: the Task Force for the Diagnosis and Treatment of Pulmonary Hypertension of the European Society of Cardiology (ESC) and the European Respiratory Society (ERS), endorsed by the International Society of Heart and Lung Transplantation (ISHLT). Eur Heart J. 2009;30(20):2493-537.

8. Duffels MGJ, Vis JC, van Loon RLE, et al. Down patients with Eisenmenger syndrome: is bosentan treatment an option? Int $\mathrm{J}$ Cardiol. 2009;134(3):378-83.

9. Vis JC, Duffels MG, Mulder P, et al. Prolonged beneficial effect of bosentan treatment and 4-year survival rates in adult patients with pulmonary arterial hypertension associated with congenital heart disease. Int J Cardiol. 2011.

10. Miyamoto S, Nagaya N, Satoh T, et al. Clinical correlates and prognostic significance of six-minute walk test in patients with primary pulmonary hypertension. Comparison with cardiopulmonary exercise testing. Am J Respir Crit Care Med. 2000;161(2 Pt 1):487-92.

11. Schuuring M, Bouma B, Cordina R, et al. Treatment of Segmental Pulmonary Artery Hypertension in Adults with Congenital Heart Disease. Int J Cardiol. 2011; In press.

12. Galiè N, Rubin L, Hoeper M, et al. Treatment of patients with mildly symptomatic pulmonary arterial hypertension with bosentan (EARLY Study). Lancet. 2008;371:2093-100.

13. Inai $K$, Nakanishi $T$, Nakazawa $M$. Clinical correlation and prognostic predictive value of neurohumoral factors in patients late after the Fontan operation. Am Heart J. 2005;150(3):588-94.

14. Schuuring MJ, Vis JC, Bouma BJ, et al. Rationale and design of a trial on the role of bosentan in Fontan patients: improvement of exercise capacity? Contemp Clin Trials. 2011.

15. Schuuring MJ, Bolmers PPM, Mulder BJM, et al. Right ventricular function declines after cardiac surgery in adult patients with congenital heart disease. Int J Cardiovasc Imaging. 2011.

16. Bond BR, Dorman BH, Clair MJ, et al. Endothelin-1 during and after cardiopulmonary bypass: association to graft sensitivity and postoperative recovery. J Thorac Cardiovasc Surg. 2001;122(2):358-64.

17. Joffs C, Walker CA, Hendrick JW, et al. Endothelin receptor subtype A blockade selectively reduces pulmonary pressure after cardiopulmonary bypass. J Thorac Cardiovasc Surg. 2001;122(2):365-70. 